\title{
Metastatic Infection by Methicillin-Sensitive Staphylococcus aureus and its Potential Association to an Unusual Humoral Immunodeficiency
}

\author{
María Cecilia Yubini ${ }^{1}$, Caterina Contreras ${ }^{1}$, María Alejandra Cerda ${ }^{2}$, Gonzalo Diaz ${ }^{2}$, \\ María Antonieta Guzmán ${ }^{3}$, Dannette Guiñez ${ }^{2}$, Rodrigo Cornejo ${ }^{2, *}$ \\ ${ }^{1}$ Faculty of Medicine, University of Chile, Santiago, 8380453, Metropolitan Region, Chile \\ ${ }^{2}$ Medicine Department, Critical Care Unit, Clinical Hospital Universidad de Chile, Santiago, 8380456, Metropolitan Region, Chile \\ ${ }^{3}$ Medicine Department, Immunology, Clinical Hospital Universidad de Chile, Santiago, 8380456, Metropolitan Region, Chile
}

Copyright $\bigcirc 2018$ by authors, all rights reserved. Authors agree that this article remains permanently open access under the terms of the Creative Commons Attribution License 4.0 International License

\begin{abstract}
Multifocal metastatic infection by Staphylococcus aureus is severe and uncommon. We describe the case of a previously asymptomatic 63 -year-old man diagnosed with complicated staphylococcal endocarditis of the mitral valve along with cervical and lumbar spondylodiscitis, and multiple pre/para-vertebral abscesses; establishing a persistent flaccid tetraparesis, neurogenic bladder and anal sphincter dysfunction. The patient overcame infection after an 8-week Cloxacillin/ Rifampicin treatment associated to multiple surgical procedures. Thereafter, immunological assessment was performed, driving to the diagnosis of selective $\operatorname{IgM}$ deficiency. We propose a physiopathological relationship between this rare dysgammaglobulinemia and such fulminant staphylococcal invasive infection; that would, perhaps, justify humoral immunity assessment in patients undergoing staphylococcemia.
\end{abstract}

Keywords Selective IgM Deficiency, Staphylococcus aureus, Metastatic Infection, Infective Endocarditis, Abscess

\section{Introduction}

Staphyloccocus aureus (S. aureus) infections have a wide spectrum of clinical presentations; from asymptomatic colonization, superficial infection, toxic-shock-syndrome, to deep metastatic infection. Although this variety of scenarios is related to the versatile virulence of this germ; certain situations as recurrence or unusual course of infection may suggest some underlying immunodeficiency. Regarding humoral immunodeficiencies, a greater risk for these infections have been noted in patients with common-variable immunodeficiency, agammaglobulinemia and B-cell leukemia; emphasizing the role of $\operatorname{IgA}$ and $\operatorname{IgG}$ in S. aureus superficial and deep infections, respectively [1].
Nevertheless, little importance has been given to selective IgM deficiency (SIgMD) upon its association to $S$. aureus infections, probably due to its low prevalence; estimated around $0.03-0.37 \%$ in general population [2-5]. We report the case of an adult man, without known risk factors, who shows-up with staphylococcemia associated to endocarditis, spondylodiscitis and multiple pre/para-vertebral abscesses, in whose assessment SIgMD is reported.

\section{Case Report}

A 63-year-old man, with history of traumatic-lumbar-fracture 8 years ago with no surgical treatment, arrives to our hospital presenting a week of fever and intense lumbar pain, poorly responsive to analgesics; developing rapidly progressive weakness of upper and lower extremities, associated to urinary incontinence and constipation. At admission he is found tachycardic (113 lpm), normotensive $(147 / 77 \mathrm{mmHg})$, with a $96 \%$ oxygen-saturation. Physical examination evidences a meso-telesystolic IV/VI mitral murmur, with no third-heart-sound and clear lungs at auscultation. Neurological exam shows an asymmetric, mainly proximal, flaccid tetraparesis; enlarged-palpable bladder, and hypotonic anal sphincter. Laboratory exams suggest an acute infection in course; which is initially assessed by a spinal CT-scan that shows multiple collections in left psoas and lumbar pre/para-vertebral muscles. A complementary MRI further denotes paravertebral collections at C3 level and L3-L4 spondylodiscitis (Figure 1). Hence, lumbar exploration and surgical drainage of the abscesses are firstly performed, in association to wide-spectrum empiric antibiotics, adjusting therapy to Cloxacillin/ Rifampicin 48 hours later; once Methicillin-Sensitive $S$. aureus positive blood-cultures are obtained. Next, lumbar (L2-L4) and cervical (C3-C6) decompressive laminectomy are executed within two surgical procedures. 


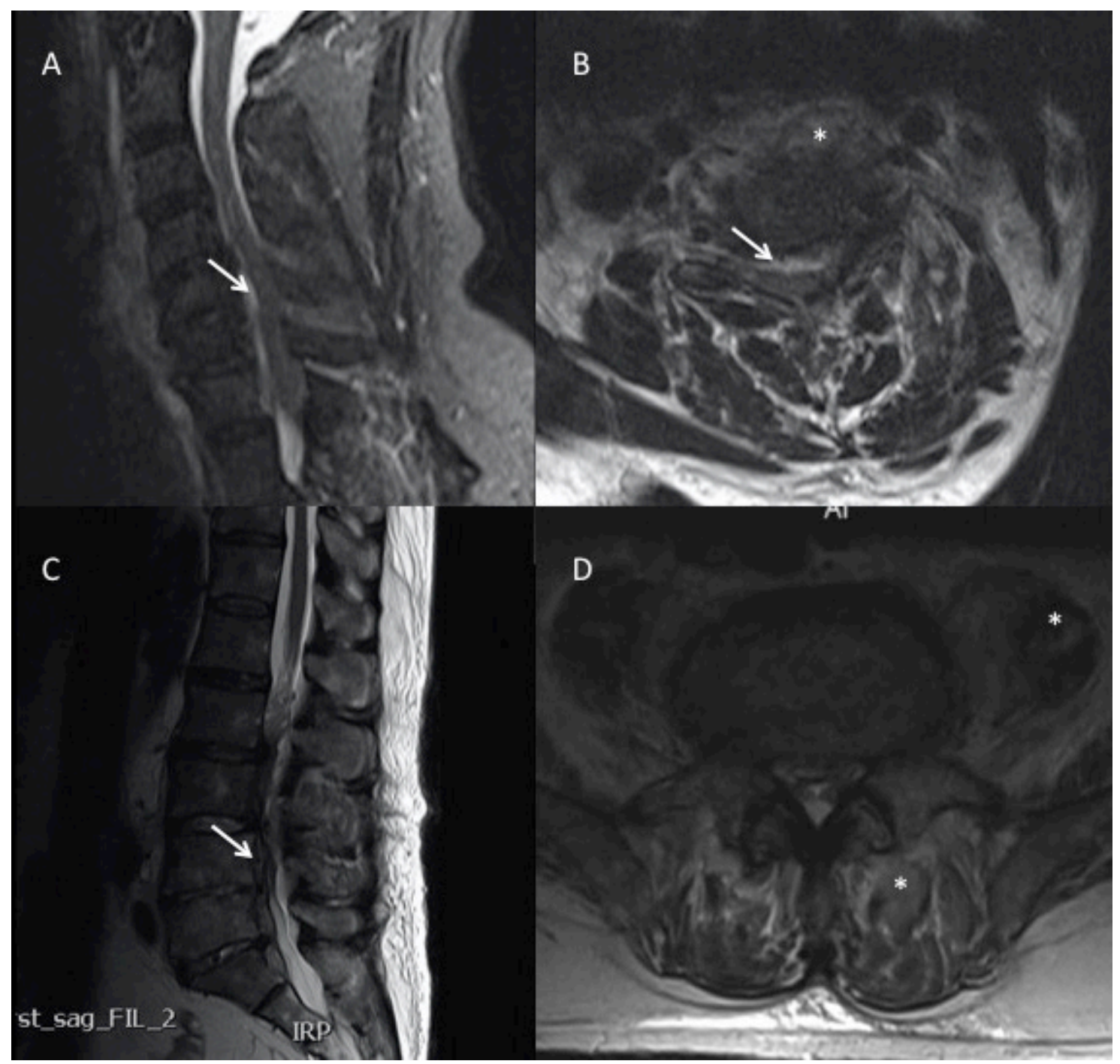

Figure 1. Magnetic resonance imaging of cervical (A y B) and lumbar (C y D) vertebral spine showing paravertebral $\left({ }^{*}\right)$ and anterior epidural (white arrows) collections, which determine an important spinal stenosis. Within lumbar levels, edema of vertebral bodies at L4 and L5 along with changes of the signal in the intervertebral disc and vertebral platforms are highly suggestive of spondylodiscitis

Assessment is followed by two-dimensional echocardiography; accusing an infective-endocarditis with severe mitral insufficiency and valve rupture. In this context, the patient undergoes mitral valve-replacement surgery.

Infection is resolved after an 8-week antibiotic therapy and surgical drainage. Unfortunately, flaccid tetraparesis persists, having a motor and sensitive level at $\mathrm{C} 4$, associated to neurogenic bladder and loss of voluntary anal sphincter control. All these neurological sequelae establish full functional-dependence for daily-life activities; therefore, the patient continues his rehabilitation at the physical-medicine department.

After overcoming the critical phase of infection, laboratory assessment for immunosuppression is done; including blood and urine electrophoresis that prove normal and many other tests (Table 1), finding exclusively and repeatedly a SIgMD.

\section{Discussion}

Metastatic infection defined as deep-seated infection including endocarditis, osteomyelitis, spondylodiscitis, and muscle or visceral abscesses; appears in $13-39 \%$ of $S$. aureus bacteremic cases. Among prognostic factors for this infection-phenotype we found: community acquisition, antibiotic treatment delay, persistent fever, persistently positive blood-cultures and the C-reactive-protein quantitative curve [6]. The unfavorable course of this severe $S$. aureus infection in an adult without known risk factors drove to SIgMD diagnosis, opening up the discussion about an eventual association between both phenomena. SIgMD is a rare dysgammaglobulinemia defined by isolated low serum-IgM levels (2 SDs below age-adjusted means) $[2,3,5]$. It was first described in 1967 , as two children debuted with fulminant meningococcal septicemia [7]. Since then, about 160 SIgMD cases have been reported, occasionally in association to other immune 
diseases $[3,8,9]$. Prevalence is estimated around $0.03-0.37 \%$ in general population, $0.07-0.26 \%$ in Immunology-\&-Allergy Clinics, $0.1-3.8 \%$ in hospitalized patients, and up to $6 \%$ within primary-immunodeficiency patients $[2-5,10]$. Apparently, there is no difference in SIgMD sex-distribution [2], however it tends to be more frequently symptomatic in men, finding a male-to-female ratio of even 11:2 [3, 5, 9, 11, 12]. Given this incomplete penetrance, an autosomal hereditary basis, perchance related to the X-chromosome, has been proposed for SIgMD etiology, in accordance with some familial case-reports and others related to congenital disorders such as Wiskott-Aldrich-Syndrome $[2,5,7,12]$. Another consistent association about genetic involvement is 22q11.2-deletion syndrome, yet it would not explain this immunodeficiency by itself [2]. Recently, the role of certain impairments on B-Lymphocyte maturation and activation processes along with Regulatory-T-Lymphocytes function have been emphasized within the SIgMD pathogenesis, which remains undefined $[1,3,13,14]$.

Table 1. Immunological assessment

\begin{tabular}{|c|c|c|}
\hline Laboratory Test & Result & Reference Range \\
\hline Hemoglobin (g/dL) & 7.3 & $13.5-17$ \\
\hline Platelet count $(/ \mu 1)$ & 351.000 & $150.000-400.000$ \\
\hline Leukocyte count $(/ \mu \mathrm{l})$ & 4.630 & \\
\hline Neutrophils (\%) & 62 & \\
\hline Monocytes (\%) & 6 & $4.000-10.000$ \\
\hline Eosinophils (\%) & 5 & \\
\hline Lymphocytes (\%) & 27 & \\
\hline $\begin{array}{c}\text { HIV-test } \\
\text { (4th gen ELISA) }\end{array}$ & Not reactive & \\
\hline \multicolumn{3}{|c|}{ Lymphocyte subsets $(\%-/ \mu 1)$} \\
\hline CD3 & $81-1104$ & $55-84 \%-690-2540 / \mu 1$ \\
\hline CD4 & $44-594$ & $31-60 \%-410-1590 / \mu 1$ \\
\hline CD8 & $32-434$ & $13-41 \%-190-1140 / \mu 1$ \\
\hline CD19 & $7-102$ & $6-25 \%-90-660 / \mu 1$ \\
\hline $\mathrm{CD} 16 / 56$ & $11-148$ & $5-27 \%-90-590 / \mu 1$ \\
\hline \multicolumn{3}{|c|}{ Serum Immunoglobulin (mg/dl) } \\
\hline IgG & 3090 & $639-1349$ \\
\hline $\operatorname{IgA}$ & 439 & $70-312$ \\
\hline IgM & 20.1 & $56-352$ \\
\hline \multicolumn{3}{|c|}{ Serum light $\&$ heavy-chain immunofixation } \\
\hline $\begin{array}{c}\text { Anti IgG } \\
\text { Anti IgA } \\
\text { Anti IgM } \\
\text { Anti-Kappa } \\
\text { Anti-Lambda }\end{array}$ & $\begin{array}{c}\text { Diffuse } \\
\text { precipitate } \\
\text { Diffuse } \\
\text { precipitate } \\
\text { No } \\
\text { precipitation } \\
\text { Diffuse } \\
\text { precipitate } \\
\text { Diffuse } \\
\text { precipitate }\end{array}$ & \\
\hline
\end{tabular}

Clinically, SIgMD can course asymptomatic in up to $50 \%$ of cases [15], as in our patient. However, it has been classically associated to recurrent infections by different agents; autoimmune diseases, immune-allergic diseases, and malignancies; mainly hematologic $[2,3,10,15]$.
Within field of infection, we found more or less severe cases of otitis media, sinusitis, bronchitis, pneumonia, septic arthritis, upper/lower urinary tract infections, meningitis, herpes zoster, pyoderma, cellulitis, erysipelas, impetigo, pulmonary/miliary tuberculosis and other mycobacterial infections [2-5, 7-12, 15-17]. Although SIgMD patients with other staphylococcal infections have been previously described [11, 12], this would be the first report of, specifically, multi-organic metastatic infection by $S$. aureus associated to SIgMD.

Features from both; host and agent, suggest a relationship potentially responsible for the clinical evolution of the described case. On one side, the immune system has developed specific mechanisms against extracellular-capsulated-bacteria like $S$. aureus; including a humoral response that neutralizes toxins and favors antibody and complement-mediated phagocytosis (opsonization), besides generating immunological memory. Ultimately, humoral immunity has attained a leading role among staphylococcal infections, as a higher predisposition for their acquisition has been recognized in patients with underlying B-cell leukemia, common-variable immunodeficiency, agammaglobulinemia or selective B-cell disorders $[1,18]$. Despite a greater importance has been usually given to $\operatorname{IgA}$ in superficial infections and to IgG in the invasive ones; it has been seen that IgM accomplishes opsonizing functions similarly to IgG, though by different molecular pathways. Studies made in staphylococcal brain abscesses murine models concluded that microglial IgM-mediated phagocytosis occurs by the activation of complement-receptor-3, independently from the IgG pathway (through $\mathrm{Fc} Y$-receptors) [19]. Furthermore, activation of B-lymphocytes in spleen and lymph-nodes depends on transmembrane-IgM, for the subsequent proliferation of lymphocyte subsets (germinal-center reaction), immunoglobulin class-switch and differentiation to high-affinity antibody (HAAb) secreting plasmocytes and memory B-Lymphocytes [20]. Then, it is inferable that a subjacent mechanism for SIgMD could be a B-lymphocyte defect, as it has already been shown in a subgroup of patients, resulting in a multifactorial deficient humoral response $[1,3,13,14]$.

On the other side, $S$. aureus has diverse well known virulence factors; classifiable as pathogenic mechanisms, antibiotic-resistance and immune-evasion. With respect to the latter, the ability to antagonize specific immunological elements, including $\operatorname{IgA}$, IgG and $\operatorname{IgM}$, has become a documented feature of $S$. aureus; identifying strategies such as bacterial intracellular-survival, immunoglobulin cleavage by several secreted proteases, and expression of super-antigens that trigger a dysfunctional humoral response. A variety of molecules have been investigated, recognizing the key role of Protein A (SpA), immunoglobulin-binding protein (Sbi), and adenosine synthase A (AdsA) [1, 18, 21]. While SpA and Sbi share 
the purpose of binding to the $\mathrm{FcY}$-domains of $\operatorname{IgG}$ with such disorientation that avoids the attachment to their receptors within neutrophils; AdsA confers an anti-inflammatory state that suppresses the innate and adaptive immune responses by the activation of adenosine receptors in diverse cells, besides allowing $S$. aureus persistence inside neutrophil phagosomes. $\mathrm{SpA}$ is the most assessed molecule, and it gets further interest concerning this case since, besides interfering with $\mathrm{IgG}$-mediated phagocytosis, it binds to VH3-receptors in B-Lymphocytes (Fab region of transmembrane-IgM) triggering the proliferation and later apoptosis of the subsets that recognized soluble SpA, regardless of its ability to induce an immune-tolerance state after combined activation of these and other receptors when $\mathrm{SpA}$ is presented together with more bacterial cell-wall antigens. Beyond this, $S$. aureus pathogenic-factors vary upon the different phenotypes of interactions with the host; determining either the condition of asymptomatic-carrier in near 20-30\% of adults, superficial infections, toxic-shock-syndrome, or deep-invasive infection $[1,21,22]$. About the last one, a four-step process has been proposed [23], by which $S$. aureus sequentially expresses specific factors, including $\mathrm{SpA}$, without which it is unable to form deep-abscesses. This sine-qua-non condition has been proven in animal models by $S$. aureus-mutant strains containing non-toxigenic, non-immunogenic $\mathrm{SpA}$, therefore not forming abscesses; additionally to the many experimental-studies focused on blocking these molecules for the assessment of anti-staphylococcal vaccines, which remains unachievable [21-23].

Based on the exposed data, the most logical association between IgM deficiency and such severe infection, is that this dysgammaglobulinemia favours the bacterial spread; as other antibody deficiencies do. Nevertheless, a valid inquiry arises when analysing this particular case; as the patient has no registry of IgM quantification prior to $\mathrm{S}$. aureus invasive disease: Could this severe infection have altered IgM levels, being the immune deficiency a consequence rather than a cause of the bacterial metastatic spread? On the attempt to answer this question it is necessary to clarify that immunological assessment, including serum IgM levels, were done once antibiotic treatment was almost over and after the infection was technically solved. Additionally, right after the first altered result, a second measurement of serum immunoglobulins was performed, finding consistently low IgM levels under $2 \mathrm{SD}$ from adjusted means with normal IgA and IgG levels, meeting the defined criteria to stablish SIgMD diagnosis [2]. Now, are we facing a primary or secondary IgM deficiency? Although secondary IgM deficiency is more common than primary SIgMD and it may appear during episodes of infection, immunosuppression treatments, chemotherapy, and/or other conditions, this secondary deficiency is usually not "selective" and, in the case of acute infection, the immunoglobulin-level variations should be transient and are expected to normalize after the infective disease is overcome [24-29]. With respect to SIgMD, there are some described associations to Celiac disease (also related to other humoral deficiencies), autoimmune diseases, atopic disorders and some haematological malignancies. However, there is not always enough evidence to stablish causality and ascribe secondary SIgMD in these cases $[30,31]$. Then, considering SIgMD as a primary condition is there any published data supporting that this disease favours invasive bacterial infections? A very interesting study from Martinot et al., assessed the proportion of immunoglobulin deficiencies within patients diagnosed with Streptococcus pneumoniae or Haemophilus influenza invasive diseases. From a total of 109 patients, $37 \quad(33.9 \%)$ had immunoglobulin deficiencies; finding a high rate of isolated IgM deficiency (13/109 patients or $11.3 \%$ ) [29]. About the mechanisms involved in this greater susceptibility to infections, it has been proposed that it may be due to an associated impaired IgG-specific antibody response to T-independent polysaccharide antigens [16], that is consistent to some observations done in mice models lacking secreted IgM and having normal isotype switch to $\mathrm{IgG}$, which have shown to be unable to control viral, bacterial and fungal infections due to an inadequate induction of a protective specific $\operatorname{IgG}$ antibody response and lack of serum IgM [25]. Besides these remarks, the contribution of IgM in the control of bacterial infections has been proven by different -animal and humanexperimental models; demonstrating a role in preventing bacterial colonization, increasing complement bactericidal activity, activating cytokine secretion and neutrophil recruitment, among other functions [32-34]. In this sense, it is easy to understand that the lack of serum IgM, as it is characteristic in patients with SIgMD, may imply an uncontrolled bacterial load, favoring systemic spread infections.

\section{Conclusions}

The exposed evidence supports a potential relationship between SIgMD and S. aureus metastatic infection so that; if the bacteria have designed specific strategies for escaping host immunity, including IgM, as a requirement for developing invasive infections; then it is reasonably considerable that the humoral response, and particularly IgM, plays a determinant role in this kind of staphylococcal infections. Thus, in SIgMD the interaction between host and agent favors the latter; expressing its intrinsic pathogenicity without an efficient humoral response that enables its clearance. Accordingly, assessment of the humoral status could be helpful among patients undergoing staphylococcemia, since such immunodeficiencies would confer a higher risk for severe metastatic infection, as it has been reported to be 
associated to other invasive bacterial infections. It is still to be defined whether SIgMD patients who experience deep-seated infections could aspire to specific therapies, beyond antibiotics and drainages, such as intravenous immunoglobulin replacement, which has already shown good results in a particular subgroup of patients $[16,35]$. Otherwise, if the development of an effective anti-staphylococcal vaccine is accomplished; this group could have a greater benefit from such achievement.

\section{Conflict of Interests}

The authors declare that they have no competing interests.

Sources of financial support: none

\section{REFERENCES}

[1] Bröker B, Holtfretera S, Bekeredjian-Ding I. Immune control of Staphylococcus aureus - Regulation and counter-regulation of the adaptive immune response. International Journal of Medical Microbiology 2014; 304: $204-214$.

[2] Louis A, Gupta S. Primary Selective IgM Deficiency: An Ignored Immunodeficiency. Clinic Rev Allerg Immunol 2014; 46(2):104-11.

[3] Goldstein M, Goldstein A, Dunsky E, Dvorin D, Belecanech G, Shamir K. Selective IgM immunodeficiency: retrospective analysis of 36 adult patients with review of the literature. Ann Allergy Asthma Immunol. 2006; 97:717730 .

[4] Cassidy JT, Nordby GL. Human serum immunoglobulin concentrations: prevalence of immunoglobulin deficiencies. J Allergy Clin Immunol 1975; 55:35-48.

[5] Entezari N, Adab Z, Zeydi M, Saghafi S, Jamali M, Kardar G, Pourpak Z.The prevalence of Selective Immunoglobulin M Deficiency (SIgMD) in Iranian volunteer blood donors. Human Immunology 2016; 77:7-11.

[6] Horino T, Sato F, Hosaka Y, Hoshina T, Tamura K, Nakaharai K, Kato T,Nakazawa Y, Yoshida M, Hori S. Predictive factors for metastatic infection in patients with bacteremia caused by methicillin-sensitive Staphylococcus aureus. Am J Med Sci 2015; 349(1):24-28.

[7] Hobbs JR, Milner RD, Watt PJ. Gamma-M deficiency predisposing to meningococcal septicaemia. Br Med J 1967; 4:583-586.

[8] Gharib A, Louis A, Agrawal S, Gupta S. Syndrome of selective $\operatorname{IgM}$ deficiency with severe $\mathrm{T}$ cell deficiency associated with disseminated cutaneous mycobacterium avium intracellulaire infection. Am J Clin Exp Immunol 2015; 4(2):15-27.

[9] Ideura G, Agematsu K, Komatsu Y, Hatayama O, Yasuo M, Tsushima K, Hanaoka M, Koizumi T, Fujimoto K, Kubo K. Selective IgM Deficiency Accompanied with IgG4
Deficiency, Dermal Complications and a Bronchial Polyp. Allergology International. 2008; 57:99-105.

[10] Hassanein H, Elbadry M. Selective immunoglobulin M deficiency in an adult with miliary tuberculosis: A clinically interesting coexistence. A case report and review of the literature. Internal Journal of Mycobacteriology 2016; 5:106-110.

[11] Yocum M, Strong D, Chusid M, Lakin J. Selective lmmunoglobulin M (IgM) Deficiency in Two lmmunodeficient Adults with Recurrent Staphylococcal Pyoderma. Am J Med 1976; 60(4):486-94.

[12] Giasuddin A, J Idoko, R Lawande. Tropical pyomyositis: is it an immunodeficiency disease? Am I Trop Med Hyg 1986; 35(6): 1231-1234.

[13] Mensen A, Krause T, Hanitsch L, Meisel C, Kleint M, Volk $\mathrm{H}, \mathrm{Na}$ I, Scheibenbogen C. Altered B-cell subsets and functional B-cell defects in selective IgM deficiency. Clin Immunol 2015; 161:96-102.

[14] Louis A, Agrawal S, Gupta S. Analysis of subsets of B cells, Breg, CD4Treg and CD8Treg cells in adult patients with primary selective IgM deficiency. Am J Clin Exp Immunol 2016; 5(1):21-32

[15] Sánchez G, De la Sen M, León R, Pinargote H, Mateo J, Zurita A, Portilla J, Sánchez R. Fenotipos clínicos asociados a la deficiencia selectiva de IgM: estudio descriptivo de 76 casos. Rev Clin Esp. 2014; 214 (Espec Congr): 1223 .

[16] Yel L, Ramanuja S, Gupta S. Clinical and Immunological features in IgM deficiency. Int Arch Allergy Immunol 2009; 150:291-298.

[17] Phuphuakrat A, Ngamjanyporn P, Nantiruj K Luangwedchakarn V, Malathum K. Selective IgM deficiency in an adult presenting with Straptococcus pneumoniae septic arthritis. J Microbiol Immunol Infect 2016; 49:150-153.

[18] Krishna S and Miller L. Innate and adaptive immune responses against Staphylococcus aureus skin infections. Semin Immunopathol 2012; 34:261-280.

[19] Weinstein J, Quan Y, Hanson J, Colonna L, Iorga M, Honda S, Shibuya K, Shibuya A, Elkon K, Möller T. IgM-Dependent Phagocytosis in Microglia Is Mediated by Complement Receptor 3, Not Fc $\alpha / \mu$ Receptor. The Journal of Immunology, 2015; 195: 000-000.

[20] LeBien T, Tedder T. B lymphocytes: how they develop and function. Blood 2008; 112:1570-1580.

[21] Kim H, Thammavongsa V, Schneewind O, Missiakas D. Recurrent infections and immune evasion strategies of Staphylococcus aureus. Curr Opin Microbiol 2012; 15:9299.

[22] Falugi F, Kim H, Missiakas D, Schneewind O. The role of protein $\mathrm{A}$ in the evasion of host adaptive immune responses by Staphylococcus aureus. mBio 2013; 4(5):e00575-13.

[23] Cheng A, Kim H, Burts M, Krausz T, Schneewind O, Missiakas D. Genetic requirements for Staphylococcus aureus abscess formation and persistence in host tissues. FASEB J. 2009; 23(10):3393-404. 
[24] Van der Burg M, Weemaes C, Cunningham-Rundles C. Stiehm's Immune Deficiencies. Academic Press, USA, 2014.

[25] Gupta S, Gupta A. Selective IgM deficiency-An underestimated primary immunodeficiency. Front. Immunol. 2017; 8:1056.

[26] Tallantyre E, Whittam D, Jolles S, Paling D, Constantinesecu C, Robertson N, Jacob A. Secondary antibody deficiency: a complication of anti-CD20 therapy for neuroinflammation. Journal of Neurology 2018.

[27] Duraisingham S, Buckland M, Dempster J, Lorenzo L, Grigoriadou S, Longhurst H. Primary vs. Secondary Antibody Deficiency: Clinical Features and Infection Outcomes of Immunoglobulin Replacement. PLoS One 2014;9(6):e100324

[28] Wirsum C, Glaser C, Gutenberger S, Keller B, Unger S, Voll R, Vach W, Ness T, Warnatz K. Secondary Antibody Deficiency in Glucocorticoid Therapy Clearly Differs from Primary Antibody Deficiency. J Clin Immunol 2016;36(4):406-12.

[29] Martinot M, Oswald L, Parisi E, Etienne E, Arggy N, Grawey I, De Briel d, Mohseni M, Federici L, Blaison G, Koebel C, Jaulhac B, hansmann Y, Christmann D. Immunoglobulin deficiency in patients with Streptococcus pneumoniae or Haemophilus influenzae invasive infections. International Journal of Infectious Diseases 2014; 19: 79 84.

[30] Chovancova Z, Kralickova P, Pejechalova A, Bloomfield M, Nechvatalova J, Vlkova M, Litzman J. Selective IgM deficiency: Clinical and laboratory features of 17 patients and a review of the literature. J Clin Immunol 2017; 37(6):559-574.

[31] Magen E, Feldman V, Joseph M, Israel H. Symptomatic secondary selective IgM deficiency in adult man with undiagnosed Celiac disease. Case Reports Immunol. 2012; 2012:684247.

[32] Boes M, Prodeus A, Schmidt T, Carroll M and Chen J. A Critical Role of Natural Immunoglobulin $M$ in Immediate Defense against Systemic Bacterial Infection. J. Exp. Med. 1998; 188(12); 2381-2386.

[33] Langereis J, Henriet S, Kuipers S, Weemaes C, Van Der Bub M, De Jonge M and Van Der Flier M. IgM Augments Complement Bactericidal Activity with Serum from a Patient with a Novel CD79a Mutation. Journal of Clinical Immunology 2018; 38:185-192.

[34] Micol R, Kayal S, Mahlaoui N, Beauté J, Brosselin P, Dudoit Y, Obenga G, Barlogis V, Aladjidi N, Kebaili K, Thomas C, Dulieu F, Monpoux F, Nové-Josserand R, Pellier I, Lambotte O, Salmon A, Masseau A, Galanaud P, Oksenhendler E, Tabone MD, Teira P, Coignard-Biehler H, Lanternier F, Join-Lambert O, Mouillot G, Theodorou I, Lecron JC, Alyanakian MA, Picard C, Blanche S, Hermine O, Suarez F, Debré M, Lecuit M, Lortholary O, Durandy A and Fischer A. Protective effect of IgM against colonization of the respiratory tract by nontypeable Haemophilus influenzae in patients with hypogammaglobulinemia. J Allergy Clin Immunol. 2012 Mar; 129(3):770-7.

[35] Goldstein M, Hilditch G, Dvorin D, Belecanech G. Immunoglobulin replacement for selective IgM immunodeficiency, bronchiectasis, and asthma. Ann Allergy Asthma Immunol 2016; 116(2):172-173. 